\title{
HYDROLYSIS OF FISH OIL BY \\ LIPASES IMMOBILIZED INSIDE POROUS SUPPORTS
}

Gloria Fernández-Lorente ${ }^{b}$, Carolina Pizarro ${ }^{a}$, Dolores López-Vela ${ }^{a}$, Lorena Betancor ${ }^{c}$, Alfonso V. Carrascosab ${ }^{b}$ Benevides Pessela ${ }^{b}$, Fernando López-Gallego and Jose M. Guisan $^{\mathrm{a} *}$

a.- Instituto de Catalisis. CSIC. Campus UAM-Cantoblanco. 28049 Madrid. Spain

b.- Instituto de Fermentaciones Industriales. CSIC. c/ Juan de la Cierva 3. 28006. Madrid. Spain

c.- IMDEA-Alimentación. Campus UAM-Cantoblanco

*To whom correspondence should be addressed

Prof. Jose M. Guisan

e-mail: jmguisan@icp.csic.es

Tel: +34915854809

Fax: +34 915854760 


\section{ABSTRACT}

A new assay was designed to measure the release of omega-3 acids (eicosapentaenoic acid (EPA) and docosahexaenoic acid (DHA)) from the hydrolysis of sardine oil by lipases immobilized inside porous supports. A biphasic system was used containing the fish oil dissolved in the organic phase and the immobilized lipase suspended in the aqueous phase. The assay was optimized by using a very active derivative of Rhizomucor miehie lipase (RML) adsorbed onto octyl agarose. Optimal conditions were: a.- an organic phase composed by $30 / 70$ (v:v) of oil in cyclohexane, b.- an aqueous phase containing $50 \mathrm{mM}$ methylcyclodextrin in $10 \mathrm{mM}$ TRIS buffer at pH 7.0. The whole reaction system was incubated at $25^{\circ} \mathrm{C}$. Under these conditions, up to $2 \%$ of the oil is partitioned into the aqueous phase and most of the $95 \%$ of released acids were partitioned into the organic phase. The organic phase was analyzed by RP-HPLC (UV detection at $215 \mathrm{~nm}$ ) and even very low concentrations (eg., $0.05 \mathrm{mM}$ ) of released omega-3 fatty acid could be detected with a precision higher than $99 \%$. Three different lipases adsorbed on octyl-agarose were compared: Candida antarctica lipase-fraction B (CALB), Thermomyces lanuginosa lipase (TLL) and RML. The three enzyme derivatives were very active. However, most active and selectivetowards polyunsaturated fatty acids (PUFAs) versus oleic plus palmitic acids (a 4-fold factor) was CALB. On the other hand, the most selective derivatives towards EPA versus DHA (a 4.5 fold factor) were TLL and RML derivatives.

Keywords: enzymatic release of omega-3 acids, selective enzymatic release of EPA, solubilization of oil by cyclodextrins. 


\section{INTRODUCTION}

The release of omega 3 fatty acids(eg. EPA and DHA) from fish oil may mean an interesting first step in the preparation of highly enriched triglycerides (70 to $90 \%$ of content in one or both polyunsaturated fatty acids, PUFAs). These triglycerides have been described as excellent functional ingredients (1). Recently, PUFAs have meant a breakthrough amongst health care professionals of the beneficial effects of omega-3 fatty acids derived from fish oils - mainly consisting of docosahexaenoic acid (DHA) plus eicosapentaenoic acid (EPA) mixtures. DHA is required in high levels in the brain and retina as a physiologically-essential nutrient to provide for optimal neuronal functioning (learning ability, mental development) and visual acuity, in the early stages of the life (2). On the other hand, EPA is considered to have beneficial effects in the prevention of cardiovascular diseases in adults $(3,4)$. In general both PUFAs have been reported useful to prevent a significant number of health disorders (5-10).

The enzymatic release of PUFAs may have important advantages over the chemical release: mild reaction conditions, absence of undesirable byproducts, etc. Futhermore, the use of immobilized lipases may have additional technological and economical advantages for the hydrolysis.

Immobilization of enzymes inside porous supports is an interesting challenge in Enzyme Biotechnology. Commercially available supports with excellent mechanical properties and high enzyme loading capacity can be selected. In this way, very active enzyme catalyst can be used in any type of industrial reactor (stirred tank, packed bed, etc). In addition, since the easy workability of porous supports, some immobilization protocols have been used to improve enzyme properties.(eg., stabilization by a very 
intense multipoint covalent immobilization (11), hyperactivation of lipases by immobilization and post-immobilization techniques (12-13), reactivation of partially inactivated lipases (14), etc.)

Hydrolysis of fish oils by lipases immobilized inside porous supports is an otustanding example of lipase application in biotechnology. This oils from marine origin are the most important source in omega 3-PUFAs in nature, hence their importance in food technology to make new food additives. In nature, soluble lipases suffer interfacial activation by oil drops. Contrarily, using immobilized lipases on porous materials, the enzyme can only hydrolyze the oil molecules partitioned into aqueous phase (Figure 1) (15-16). However, this limitation is compensated by the plethora of advantages that immobilization gives to proteins in order to be used in industrial biotransformations. From a practical point of view, immobilized lipases can be re-used for a number of reaction cycles and lipases (inside a porous structure) cannot be inactivated by hydrophobic interfaces present in strongly stirred reactors (e.g., oil interfaces, solvent interfaces, small bubbles of oxygen, etc.).

On the other hand, a rapid evaluation of the intrinsic properties of new lipases on these oily substrates (activity, stability, selectivity) may be directly tested using immobilized derivatives even before any previous purification process. Developing this partial but very rapid and highly sensitive assay for such purposes may become of great interest.

Using biphasic systems (water / immiscible solvents) may be an interesting for the above mentioned assays. Oil will be partitioned into the organic phase and the enzymatic catalyst will be in the aqueous phase only acting on isolated oil molecules 
that are partitioned into the aqueous phase. Pure oils are very viscous and difficult to work with, but mixtures oil/organic solvents (eg. cyclohexane) could simplify the reaction design. In the case of fish oil, their high content in PUFAs (as acyl chains forming part of triacylglycerol in the oil) could facilitate the aqueous/organic partition of oil molecules. Additionally, released PUFAs could be easily detected by RP-HPLC with UV detectors.

\section{MATERIALS AND METHODS}

\section{Materials.}

Lipases from CAL-B (Novozym 525L), Rizomucor miehei (Palatase) and Thermomyces lanuginose (Lypozyme TL) were generously donated by Novozymes (Denmark). Randomly Methylated BCD (TRMB-T) (methylcyclodextrin) was layed in Cyclodextrin Resource, CTD Inc. (High Springs, Florida, USA). Triton X-100, p-nitrophenyl butyrate (pNPB), docosahexaenoic acid (DHA) and eicosapentaenoic acid (EPA) were obtained from Sigma Chemical Co. (St. Louis, USA). Octyl sepharose ${ }^{\mathrm{TM}} \mathrm{CL}-4 \mathrm{~B}$ was purchased from GE Healthcare (Uppsala, Sweden). Sardine Oil was donated by BTSA, Biotecnologías Aplicadas, S.L (Madrid. Spain). Other reagents and solvents were of analytical or HPLC grade. 


\section{Enzyme activity assay}

It was performed by measuring the increase in absorbance at $348 \mathrm{~nm}$ produced by the release of $p$-nitrophenol in the hydrolysis of $0.4 \mathrm{mM}$-nitrophenyl butyrate (pNPB) in $25 \mathrm{mM}$ sodium phosphate at $\mathrm{pH} 7$ and $25 \mathrm{C}$. To initialize the reaction, $0.1 \mathrm{~mL}$ of lipase solution or suspension was added to $2.5 \mathrm{~mL}$ of substrate solution. An international unit of pNPB activity is defined as the amount of enzyme necessary to hydrolyze $1 \mu \mathrm{mol}$ of $\mathrm{pNPB} / \mathrm{min}$ (IU) under the above described conditions.

\section{Immobilization of lipases on octyl-agarose supports}

$10 \mathrm{~g}$ of octyl-agarose were added to $900 \mathrm{~mL}$ of $5 \mathrm{mM}$ sodium phosphate buffer at $\mathrm{pH} 7$ containing $250 \mathrm{mg}$ of protein and the suspension was incubated at $25 \mathrm{o} \mathrm{C}$. A blank of each soluble enzyme with the same concentration was incubated under the same experiment conditions. The activity of the blanks was fully preserved for 4 hours. On the contrary, the activity of the supernatant of the immobilization suspension decreases down to $5 \%$. That is, in all cases, more than $95 \%$ of the enzyme was immobilized. After $4 \mathrm{~h}$, the immobilized preparation was filtered and washed with distilled water. The activity of immobilized derivatives was very low because of mass transfer limitations. The derivatives were broken (to reduce particle size) by strong magnetic stirring. After this treatment the recovered activities (towards pNPB) were 120, 1000 and $2000 \%$ for CALB, RML and TLL. The same hyperactivation was also observed for very low loaded derivatives (eg., $0.25 \mathrm{mg}$ of lipase per gram of support) 
and this hyperactivation is due to interfacial activation of lipases on hydrophobic supports $(12,17,18)$.

\section{Hydrolysis of Sardine Oil}

The hydrolysis of sardine oil was performed in an organic and aqueous two-phase system. The procedure was as follows: $5 \mathrm{~mL}$ of cyclohexane, $5 \mathrm{~mL}$ of tris buffer $(0.1 \mathrm{M})$ $\mathrm{pH} 7$, and $0.5 \mathrm{~mL}$ of sardine oil were placed in a reactor and pre incubated at $25 \circ \mathrm{C}$ for $30 \mathrm{~min}$. The reaction then was initiated by adding different amounts (from 0.1 up to 1 g) of lipase derivative and the reaction suspension was stirred at $150 \mathrm{rpm}$. A pH-stat Mettler Toledo DL50 graphic was used to maintain constant the $\mathrm{pH}$ value during the reactions. The concentration of free fatty acids was determined at various times by HPLC-ELSD and HPLC-UV methods.

\section{Analysis of Polyunsaturated free fatty acids (PUFAS) by HPLC-UV}

After a given time, aliquots of $0.1 \mathrm{~mL}$ of organic phase were withdrawn and dissolved in $0.8 \mathrm{~mL}$ of acetonitrile. The unsaturated fatty acids produced were analyzed by RPHPLC (Spectra Physic SP 100 coupled with an UV detector Spectra Physic SP 8450) using a Kromasil C8 $(15 \mathrm{~cm} \times 0.4 \mathrm{~cm})$ column. Products were eluted at a flow rate of 1.0 $\mathrm{mL} / \mathrm{min}$ using acetonitrile-10 mM ammonium TRIS buffer at $\mathrm{pH} 8(70: 30, \mathrm{v} / \mathrm{v})$ and UV detection performed at $215 \mathrm{~nm}$. The retention times for the unsaturated fatty acids were: $9.4 \mathrm{~min}$ for eicosapentaenoic acid (EPA) and $13.5 \mathrm{~min}$ for docosahexaenoic acid 
(DHA). These PUFAs enzymatically produced were compare with their corresponding pure commercial standars.

\section{Analysis of free fatty acids by HPLC-ELSD}

After a given time, aliquots of $0.1 \mathrm{~mL}$ of organic phase were withdrawn and dissolved in $0.8 \mathrm{~mL}$ of propanol. The fatty acids produced were analyzed by RP-HPLC (Spectra Physic SP 100 coupled to a ELSD 2000 evaporative laser light scattering detector). $\mathrm{N}_{2}$ was used as the nebulising gas at a flow of $3 \mathrm{~mL} / \mathrm{min}$, and a nebulising temperature of 30ㄷ. A Kromasil C18 $(25 \mathrm{~cm} \times 0.4 \mathrm{~cm})$ column was used and a gradient elution was performed using solvent A (acetonitrile/water, 98:2, v/v) and solvent B (acetonitrile/propanol, $95: 5, \mathrm{v} / \mathrm{v}$ ) with the following 22 min gradient: 0-6.5 min: 100\% A; 6.5-7.5 min: $50 \%$ A; 7.6-15 $\min : 100 \%$ B; $15.1 \mathrm{~min}-18 \mathrm{~min}: 50 \%$ B. $18.1-22 \mathrm{~min}$ : $100 \% \mathrm{~A}$. The total flow rate was set to $1 \mathrm{~mL}$ per minute. The retention times for the

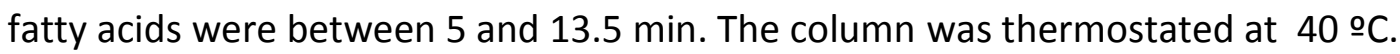




\section{RESULTS AND DISCUSSION}

\section{1.- Detection of traces of PUFAs by using isocratic RP-HPLC with UV detection.}

Polyunsaturated fatty acids (PUFAs) exhibited a high absorbance at low wavelengths in the UV region. EPA exhibited a very high absorbance at 210, 215 and $220 \mathrm{~nm}$ (Table 1). A wavelength of 215 was selected in order to minimize interferences by impurities present in sardine oil samples. By using pure commercial EPA and DHA different standard solutions with different concentration of each omega-3 fatty acids in cyclohexane were prepared. $20 \mu \mathrm{l}$ samples of each standard solution were analyzed by RP-HPLC with UV detection at a constant $1 \mathrm{ml} / \mathrm{min}$ flow rate. Integration areas were perfectly proportional to PUFA concentrations (Figure 2). The absorbance of DHA was a $50 \%$ higher than such one from EPA. UV-HPLC chromatograms of aliquots of the organic phase of the reaction mixture were compared to the corresponding calibration curves and hence the rates of hydrolysis (EPA+DHA) and the EPA/DHA ratios could be easily calculated

DHA exhibited a similar (a $50 \%$ higher) absorbance at $215 \mathrm{~nm}$ (Table 2). On the contrary, a similar concentration of oleic acid absorbed around $1 \%$ in comparison to PUFAS. Besides, the polyunsaturated structure of PUFAs may them less apolar and hence the retention times in RP-HPLC under isocratic conditions are relatively low. (Table 3).

Therefore, using an isocratic HPLC under the previously mentioned conditions, a rapid, easy and highly precise analysis of omega 3 fatty acids released by enzymatic hydrolysis of fish oil could be designed. For instance, when analyzing a sample of 0.1 $\mathrm{mM}$ DHA utilizing a $1 \mathrm{ml} / \mathrm{min}$ flow the peak area was very high (over $4800000 \pm 10000$ 
units with a very low baseline noise) (Figure 2). On the other hand, sardine oil was not spontaneously hydrolyzed at $\mathrm{pH} 6.0$ for 24 hours. Thus, EPA and DHA conversions lower than $0.2 \%$ in 24 hours (concentration of $0.05 \mathrm{mM}$ of PUFAs in the organic phase) could be easily detected. A rapid preliminary evaluation of very lowly active immobilized lipases could be performed by this protocol. In addition to that, the enzyme selectivity towards either on or the other PUFA (EPA/DHA ratio) can also be measured using this protocol because of the high resolution to separate both EPA and DHA using HPLC.

\section{2.- Use of an isocratic HPLC with a light scattering detector for the detection} of traces of fatty acids.

A "light scattering" detector (ELSD) was used to analyze the ratios between polyunsarurated fatty acids and the abundant oleic and palmitic acid also present in fish oil (19). The detection of oleic and palmitic acid (at high concentrations, eg., 0.5-1 $\mathrm{mM}$ ) was much more sensitive using this detector than the UV one and hence they were easily compared to the PUFAS. Now, a much more apolar mobile phase was used in order to decrease the retention time of oleic and palmitic acids (RT of 9 minutes approximately). Anyway, representation of the absorbance versus concentration follows a complex sigmoidal trend and hence calibration curves have to be used to calculate the exact concentration of each fatty acid (Figure 3). (20) 


\section{3.- Design of a biphasic reactor water-cosolvent for the hydrolysis of fish oil using lipases immobilized on porous supports.}

Oils are very viscous and difficult to handle (they become adsorbed on the walls of the containers, on moderately hydrophobic supports or on the stirring devices). Thus, in order to make oil manipulation feasible (especially at low oilconcentrations) the biphasic systems water-immiscible cosolvents (eg., cyclohexane) was approached. Firstly, it was proposed 1:1 ratio aqueous/ organic phase where the aqueous phase contained the catalyst (lipase) immobilized on porous structures (eg., agarose gels). In this case, the enzyme inside the porous could only hydrolyze the oil molecules present in the aqueous phase of the system.

The biphasic system and the reaction conditions were optimized by using a very active RML derivative containing $5 \mathrm{mg}$ of RML adsorbed per gram of octyl agarose (4\%) and showing 10-fold hyperactivation due to stabilization of active (open) form of the lipase (16).

\section{a.- Partioning of the released fatty acids.}

The partition of a DHA standard in a biphasic system 1:1 at different pHs was studied. In a wide range of $\mathrm{pHs}(\mathrm{pH} 5-8)$ the non ionized acid was mainly found in the organic phase. Similar values were obtained with EPA. . Since it has been reported for PUFAs a pK 9-10, (21) pH 7 seems to be suitable to perform the hydrolysis of fish oils with a number of lipases idependently of their corresponding optimal pHs. In this sense, the product to analyze (PUFAs) can be directly withdrawn from the organic phase. Another advantage of this system is the low concentration of released acids in the aqueous 
phase which would avoidproduct inhibitions issues and/or saponification processes even at high hydrolytic conversions.

\section{b.- Optimization of the reaction conditions.}

The composition of the aqueous phase was also optimized (Tables 3,4) observing two highlightable effects; low buffer concentration contributed to increase the reaction rate and added methylcyclodextrin to the aqueous phase had a positive effect in the hydrolytic activity. Considering those two beneficial effects induced by both low $\mathrm{pH}$ and methylcyclodextrin, lipase activity increased from $3.67 \mathrm{U}$ at high buffer concentrations to $20.14 \mathrm{U}$ at low ones. . This activity improvement seems to be related to a better partition of the oil in the aqueous phase from $0.15 \%$ in $100 \mathrm{mM}$ TRIS up to $2 \%$ in $20 \mathrm{mM}$ TRIS and $50 \mathrm{mM}$ methylcyclodextrin. The use of cyclodextrins to increase the solubility in water of apolar compounds has been widely utilized $(22,23)$.

On the other hand, at higher $\mathrm{pH}$ values like the activity the PUFAs type selectivity was increased because the higher EDA/DHA ratio values (Table 5). This insight migh be a intrinsic property from one particular lipase (RML in this case). This fact is hard to contrast in the literature because most of lipases have been studied at $\mathrm{pH} 7$ in this biotransformation because of the activity/stability binomial. Here we have studied this differential enzyme behavior in terms of selectivity in function of media $\mathrm{pH}$, opening a new framework to study the effect of reaction $\mathrm{pH}$ on selectivity of many lipases. 
The effect of oil concentration in the organic phase on the hydrolytic activity was also tested. From this study is observed that concentration of up to $50 \%$ of fish oil rose the lipase activity, likely because of the higher concentration of oil in the aqueous phase, increasing the substrate availability to be used by the lipase in that phase. (Table 6). Optimal reaction conditions were reached at $30 \%$ of fish oil, keeping low viscosity, making oils easier to work with at the same time than the lipase showed a high hydrolytic activity. Under these optimal conditions the rate of reaction was proportional to the amount of catalyst used (Figure 4).

\section{c.- Hydrolysis of sardine oil using three different immobilized lipases.}

Using octil-agarose derivatives of three different lipases the hydrolysis of sardine oil under optimal reaction conditions was studied. Beside to HPLC-UV analysis to follow the rate of PUFAs release and the selectivity EPA/DHA, HPLC-light scattering analysis was carried out to determine lipase selectivity towards either unsaturated or saturated fatty acids. RML andTLL were the most selective enzymes towards EPA regarding to DHA ( EPA/DHA ratio of 4.5). Unlikely, CALB did have low EPA/DHA ratio but high unsaturated/saturated (oleic or palmitic acids) ratio (around 4), showing itself as a very selective lipase towards unsaturated (EPA or DHA) acyl chains although it is not able to selectively hydrolyze different unsaturated acyl chains as DHA and EPA.

In this paper the interfacial activation is directly promoted on the support (12) and the possible inactivation by hydrophobic interfaces was avoided because the protection of immobilized enzymes inside porous structures $(24,25)$. In addition to this hyperactivation on hydrophobic supports, other hyperactivation protocols during and after immobilization have been reported (26). 


\section{CONCLUSIONS}

The optimal conditions for sardine oil hydrolysis by lipases immobilized on porous supports were a biphasic system at $25 \mathrm{C}$ where organic phase was composed by a 30/70 mixture of oil and cyclohexane and aqueous phase contained $50 \mathrm{mM}$ methylcyclodextrin in $10 \mathrm{mM}$ TRIS buffer at $\mathrm{pH}$ 6.0.. Under these conditions any derivative of any lipase (even immobilized from crude extracts) can be easily assayed: conversions as low as $0.2 \%$ of hydrolysis in 24 hours could be easily detected. In addition, the initial rate of PUFA hydrolysis (release of DHA and EPA) and the selectivity of the hydrolysis (EPA/DHA ratio)could be easily calculated.

The use of fish oil as substrate has a great practical interest because of the PUFAs releasing, being this the first step to synthesize new key functional ingredients (triglycerides highly enriched in PUFAs). From a more basic point of view, hydrolysis of fish oil (e.g. from sardine) may also be a very good test of lipolytic activities of a number of lipases: a.- sardine oil has a high percentage of the main types of fatty acids (PUFAs, monounsaturated and saturated); b.- TLL, CALB and RML (an very likely a number of other lipases) hydrolyze all kinds of fatty acids within the same order of magnitude and thus releasing of PUFAs can provide a fair semi-quantitative measurement of the whole lipolytic activity of different enzymes; c.- PUFAs could be easily detected by UV-HPLC (at $215 \mathrm{~nm}$ ) with a very high sensitivity and this fact would allow a simple test of even poorly active derivatives (eg., new crude extracts) at very low conversion values (eg., $0.2 \%$ ) for a long time (eg., 24 hours), d.- at higher conversions and using a slightly more complex system (light scattering - HPLC) the release of saturated and monounsaturated fatty acids could also been easily detected. 


\section{FIGURE LEGENDS}

Figure 1.- Some special features of hydrolysis of oils in strongly stirred biphasic systems catalyzed by lipases immobilized on porous supports. A.- Immobilized lipase is only acting on fully water soluble oil molecules. B.- There is a low partition between oil in the organic phase and oil in the aqueous phase. C.- Gas bubbles promoted by strong stirring are not able to penetrate inside the porous structure of the catalyst. D.Drops of organic phase are not able to penetrate inside the porous structure of the catalyst.

Figure 2.- UV response of EPA and DHA at different concentrations. Experimental conditions are described in Method. Response is expressed as Absorbance Units observed for the corresponding peaks in the RP-HPLC chromatograms. Injected samples: $20 \mu \mathrm{l}$ of solutions with different concentrations (from 0.125 to $2 \mathrm{mM}$ ) of PUFAs.

Figure 3.- "Light-scattering" response of EPA and DHA at different concentrations. Experimental conditions are described in Methods. Response is expressed as Absorbance Units observed for the corresponding peaks in the RP-HPLC chromatograms. Injected samples: $20 \mu \mathrm{l}$ of solutions with different concentrations of PUFAs (from 0.125 to $3 \mathrm{mM}$ ). 
Figure 4.- Effect of the amount of immobilized lipase on hydrolytic rates. Experimental conditions are described in Methods. Activity is expressed as $\mu$ mols of PUFAS (EPA plus DHA) released per minute. Amount of immobilized lipase is expressed as weight of catalyst added to $10 \mathrm{~mL}$ of biphasic reactor (from 0.1 to 1 grams of biocatalyst).

\section{REFERENCES}

(1) Fernandez L, Banuelos O, Zafra A, Ronchel C, Perez-Victoria I, Morales JC, Velasco, J, Adrio JL (2008) Alteration of substrate specificity of Galactomyces geotrichum BT107 lipase I on eicosapentaenoic acid-rich triglycerides. Biocatal. Biotransform. 26: 296305

(2) Heird WC (2001) The role of polyunsaturated fatty acids in term and preterm infants and breastfeeding mothers. Pediatr. Clin. North Am. 48: 173-188

(3) Demaison L, Moreau D (2002) Dietary n-3 polyunsaturated fatty acids and coronary heart disease-related mortality: A possible mechanism of action. Cell. Mol. Life Sci. 59:. 463-477

(4) Saremi A, Arora R (2009) The utility of omega-3 fatty acids in cardiovascular disease. American Journal of Therapeutics 16: 421-436 
(5) Antypa N, Van Der Does AJW, Smelt AHM, Rogers RD (2009) Omega-3 fatty acids (fish-oil) and depression-related cognition in healthy volunteers. J. Psychopharmacol. 23: $831-840$.

(6) Bougnoux P, Hajjaji N, Maheo K, Couet C, Chevalier S (2010) Fatty acids and breast cancer: Sensitization to treatments and prevention of metastatic re-growth. Prog. Lipid Res. 49: 76-86

(7) Montgomery P, Richardson AJ. (2008) Omega-3 fatty acids for bipolar disorder. Cochrane Database of Systematic Reviews, 2

(8) Siddiqui RA, Harvey KA, Zaloga GP (2008) Modulation of enzymatic activities by n-3 polyunsaturated fatty acids to support cardiovascular health. J. Nutr. Biochem. 19: 417-437.

(9) GISSI-HF Investigators (2008) Effect of n-3 polyunsaturated fatty acids in patients with chronic heart failure (the GISSI-HF trial): a randomised, double-blind, placebocontrolled trial. The Lancet 372: 1223-1230

(10) Riediger ND, Othman RA, Suh M, Moghadasian MH (2009) A Systemic Review of the Roles of n-3 Fatty Acids in Health and Disease. J. Am. Diet. Assoc. 109: 668-679

(11) Mateo C, Palomo JM, Fue -Gallego F, Pessela BCC, Hidalgo A, , JM (2006) Glyoxyl agarose: A fully inert and hydrophilic support for immobilization and high stabilization of proteins. Enzyme Microb. Technol. 39: 274-280 
(12) Bastida A , JM

(1998) A single step purification, immobilization, and hyperactivation of lipases via interfacial adsorption on strongly hydrophobic supports. Biotechnol. Bioeng. 58: 486493

(13) Godoy CA, Fernández-Lorente G, Felice M, De las Rivas B, Guisán JM, Palomo JM (2010) Dramatic increase in activity of immobilized derivatives of lipase from Geobacillus thermocatenolatus. Symergistic effect of different additives and sitedirected chemical modification. Enzyme Microb. Technol. Submitted

(14) Rodrigues RC, Bolivar JM, Volpato G, Filice M, Godoy C, Fernandez-Lafuente R, Guisan JM (2009) Improved reactivation of immobilized-stabilized lipase from Thermomyces lanuginosus by its coating with highly hydrophilic polymers. J. Biotechnol. 144: 113-119

(2002) Interfacial adsorption of lipases on very hydrophobic support (octadecyl-Sepabeads): Immobilization, hyperactivation and stabilization of the open form of lipases. J. Mol. Catal. B: Enzym. 19: 279-286

(1998) Immobilization of lipases by selective adsorption on hydrophobic supports Chem. Phys. Lipids 93: 185-197 
(17) Aucoin MG, Erhardt FA, Legge RL. (2004) Hyperactivation of Rhizomucor miehei Lipase by Hydrophobic Xerogels. Biotechnol. Bioeng. 85: 647-655

(18) Fernandez-Lorente G, Cabrera Z, Godoy C, Fernandez-Lafuente R, Palomo JM, Guisan JM (2008) Interfacially activated lipases against hydrophobic supports: Effect of the support nature on the biocatalytic properties. Process Biochemistry 43: 1061-1067

(19) Gámez-Meza N, Higuera-Ciapara I, Calderon De La Barca AM, Vázquez-Moreno L, Noriega-Rodríguez J, Angulo-Guerrero O. (1999) Seasonal variation in the fatty acid composition and quality of sardine oil from Sardinops sagax caeruleus of the Gulf of California. Lipids 34: 639-642

(20) Rombaut R, De Clercq N, Foubert I, Dewettinck K (2009) Triacylglycerol analysis of fats and oils by evaporative light scattering detection.

J. Am. Oil Chem. Soc. 86:19-25

(21).- Kanicky, J.R., Shah, D.O.Effect of degree, type, and position of unsaturation on the pKa of long-chain fatty acids. (2002) J. Colloid Interface Sci., 256 (1), pp. 201-207.

(22) Loftsson T, Brewster ME (1996) Pharmaceutical applications of cyclodextrins. 1. Drug solubilization and stabilization. J. Pharm. Sci. 85:1017-1025

(23) Strickley RG (2004) Solubilizing Excipients in Oral and Injectable Formulations. Pharm. Res. 21:201-230 
(24) Bes MT, Gomez-Moreno C, Guisan JM, Fernandez-Lafuente R. (1995) Selective oxidation: Stabilisation by multipoint attachment of ferredoxin NADP+ reductase, an interesting cofactor recycling enzyme. J. Mol. Catal. A: Chem. 98: 161-169

(25) Betancor L, López-Gallego F, Hidalgo A, Alonso-Morales N, Fuentes M, FernándezLafuente R, Guisán JM (2004) Prevention of interfacial inactivation of enzymes by coating the enzyme surface with dextran-aldehyde. J. Biotechnol. 110: 201-207

(26) Godoy C, de las Rivas B, Filice M, Fernández-Lorente G, Guisan JM, Palomo, JM (2009) Enhanced activity of an immobilized lipase promoted by site- directed chemical modification with polymers. Process Biochem. In press. doi: 10.1016/j.procbio.2009.11.014 